\title{
A Rapidly Cooling Neutron Star
}

\section{Astrophysicists have found the first direct evidence for the fastest neutrino-emission mechanism by which neutron stars can cool.}

\section{by James M. Lattimer*}

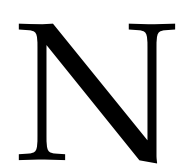

eutron stars are formed in supernova explosions of stars too massive to become white dwarfs. In such supernovae, the hot remnant formed by the collapse of the stellar core is so dense that most protons and electrons combine to make neutrons. By observing how neutron stars cool, researchers hope to understand how matter behaves under extreme conditions-densities exceeding those of atomic nuclei and temperatures that can reach hundreds of billions of kelvin. Now, Edward Brown at Michigan State University, East Lansing, and co-workers have investigated the cooling history of a neutron star that is part of a binary system in our Galaxy. By analyzing the star's x-ray emissions in the quiescent periods that followed two periods of x-ray outbursts ending in 2001 and 2016, the team found evidence of a particularly fast neutrino cooling mechanism, called the direct Urca process [1]. This process is believed to occur early in the lives of all neutron stars and may operate in later stages of a few neutron stars, but it has never been directly observed.

In a newly born neutron star, neutrinos are temporarily trapped in the opaque stellar core, but they diffuse out in a matter of seconds, leaving most of their energy to heat the matter in the core to more than 500 billion kelvin. Over the next million years, the star mainly cools by emitting more neutrinos. The direct Urca process, which is thought to dominate the initial stage of this cooling, was first proposed by physicists Mário Schenberg and George Gamow. (The mechanism is named for a casino in Rio, where Schenberg allegedly said to Gamow: "The energy in a supernova must disappear... as quickly as the money at the roulette table.") In direct Urca processes involving nucleons, thermally excited neutrons undergo beta decay to release protons, electrons, and antineutrinos, a reaction denoted $n \rightarrow p+e^{-}+\bar{v}_{e}$, while thermally excited protons undergo the reverse process and generate neutrons, positrons, and neutrinos, or $p \rightarrow n+e^{+}+v_{e}$. The neutrinos carry away energy as they escape from the star, and as the star cools, the number of thermally excited nucleons drops.

*Department of Physics and Astronomy, Stony Brook University, Stony Brook, NY 11794, USA

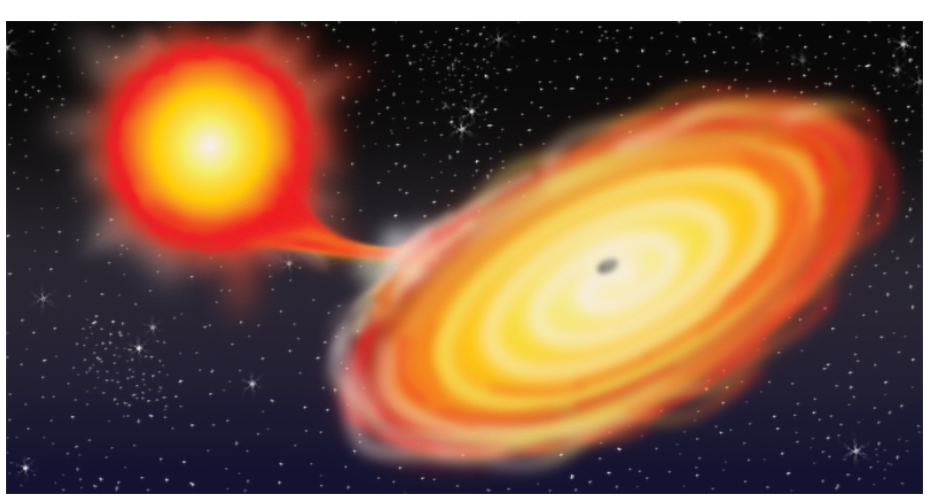

Figure 1: A neutron star in a binary system can accrete material from its companion star-a process usually associated with intense x-ray outbursts. By analyzing the outburst-quiescence cycles of a binary system in our Galaxy, Brown et al. found evidence of a fast cooling mechanism known as the direct Urca process [1]. (APS/Carin Cain)

Within a minute, the direct Urca process would allow the temperature of the stellar core to plummet to about 1 billion kelvin. But once this "low" temperature is reached, most neutron stars wouldn't have enough thermally excited nucleons to continue to support the direct Urca process because the process can no longer simultaneously conserve energy and momentum. Calculations show that to conserve momentum, the direct Urca process requires a minimum fractional proton concentration $\left(Y_{p}\right)$ of $1 / 9$ [2]. The proton fraction in a neutron star below 1 billion kelvin is determined by the so-called nuclear symmetry energy, which quantifies the change in energy occurring when protons are converted into neutrons. For typical neutron star densities, which are close to those of atomic nuclei $\left(\rho_{s}=2.3 \times\right.$ $\left.10^{17} \mathrm{~kg} / \mathrm{m}^{3}\right)$, the symmetry energy sets $Y_{p}$ at about $1 / 25$, which would stop the direct Urca process. The next available cooling mechanisms are "modified" Urca processes, which, at these temperatures, are a million times slower than the direct nucleon Urca process. As a consequence, after the first few minutes, most neutron stars cool relatively slowly and remain visible as $\mathrm{x}$-ray sources for up to a million years.

However, there may be exceptions. The nuclear symmetry energy increases with density, and it is possible that $Y_{p}$ is larger than $1 / 9$ in sufficiently massive neutron stars. Thus such stars could still undergo enhanced neutrino cooling via 
direct Urca processes. This possibility will depend both on how the symmetry energy scales with density-which is difficult to determine at high density - and on the maximum density in the star, which depends on its mass.

To test for the rapid cooling associated with the direct Urca process, researchers typically determine the temperatures and ages of isolated, thermally emitting neutron stars. By comparing a star's temperature with predictions from theoretical models, they can infer whether rapid cooling occurred in the star's early life. These indirect tests previously suggested that a handful of stars might have undergone enhanced cooling [3]. However, more recently, researchers have realized that another test is possible, based on observing neutron stars in binary systems. A neutron star in a binary may accrete material from its companion star-a process that typically generates $\mathrm{x}$-ray outbursts. During accretion, the stellar core's temperature is determined by the balance between the heating due to accretion and the cooling due to neutrino emission. In the quiescent period following accretion, the surface temperature can be used to infer the core temperature and thereby determine the cooling rate. Up until now, two binary systems [4, 5] have shown hints of enhanced cooling. In these studies, however, the evidence for enhanced cooling was indirect because the stars were already too dim for their thermal emission to be detected-only upper limits to their temperatures were inferred from observations

Brown and colleagues obtained, for the first time, direct evidence of a neutron star in the act of cooling at a rate consistent with the direct Urca process. The evidence comes from the study of a neutron star that is a member of the binary MXB 1659-29 (Fig. 1). MXB 1659-29 underwent outbursts that ended in 2001 and 2016, providing the chance to observe the cooling of the neutron star during the subsequent quiescent periods. The authors analyze the temporal history of the luminosity and temperature of the source, assuming that the outburst-quiescence cycle observed to date represents the long-term behavior of the star. Under this assumption, they conclude that the cooling observed in the star's history can only be explained if the direct Urca process is active in about $1 \%$ of the stellar core. Although other processes could, in principle, explain the observed enhanced cooling, the authors conclude that they are disfavored by the data [6].

These results are potentially very important for our understanding of dense matter. The direct Urca process requires both unpaired nucleons and a sufficiently large proton fraction in at least part of the star's core. From their estimate of the amount of unpaired nucleons in MXB 1659-29's neutron star, the researchers were able to derive constraints on the heat capacity of the star's core. They were also able to constrain the critical temperature of the superfluid state that's thought to form in the core. Such results will provide important consistency checks for dense-matter models.
What's more, the proton fraction can give information on the nuclear symmetry energy, in particular, on its density dependence. Until now, such information has been restricted by nuclear experiments to densities below those of atomic nuclei, while at the center of neutron stars, the densities can be 5 times higher. The authors estimate that the mass of MXB 1689-29's neutron star is approximately 0.03 solar masses larger than the threshold mass for the direct Urca process to occur. If the star's actual mass could be accurately determined, the value of the nuclear symmetry would be quantified at high densities for the first time.

Studies like that carried out by Brown et al. will allow researchers to probe enhanced cooling in more neutron stars and to connect such observations to the fundamental properties of dense nuclear matter. Investigating the binaries analyzed in Refs. [4] and [5] might be particularly illuminating. According to those studies, there should be more enhanced cooling in those neutron stars than in MXB 1659-29, suggesting that the stars are correspondingly more massive and thus denser. If enhanced cooling is confirmed, observations may provide experimental constraints on the symmetry energy at even higher densities than in MXB 165929.

This research is published in Physical Review Letters.

\section{REFERENCES}

[1] E. F. Brown, A. Cumming, F. J. Fattoyev, C. J. Horowitz, D. Page, and S. Reddy, "Rapid Neutrino Cooling in the Neutron Star MXB 1659-29," Phys. Rev. Lett. 120, 182701 (2018).

[2] J. M. Lattimer, C. J. Pethick, M. Prakash, and P. Haensel, "Direct URCA Process in Neutron Stars," Phys. Rev. Lett. 66, 2701 (1991).

[3] D. Page, J. M. Lattimer, M. Prakash, and A. W. Steiner, "Neutrino Emission from Cooper Pairs and Minimal Cooling of Neutron Stars," Astrophys. J. 707, 1131 (2009).

[4] P. G. Jonker, D. Steeghs, D. Chakrabarty, and A. M. Juett, "The Cold Neutron Star in the Soft X-Ray Transient 1H 1905+000," Astrophys. J. Lett. 665, L147 (2007).

[5] C. O. Heinke, P. G. Jonker, R. Wijnands, C. J. Deloye, and R. E. Taam, "Further Constraints on Thermal Quiescent X-Ray Emission from SAX J1808.4-3658," Astrophys. J. 691, 1035 (2009).

[6] The observed enhanced cooling could be due to different reactions, such as reactions involving pion or kaon condensates, unpaired quark, or hyperons. However, even the most rapid of these reactions, which involve hyperonic processes, would lead to neutrino emission rates that are at least several times smaller than the direct nucleon Urca process. To account for the observed rate of cooling, a correspondingly larger fraction of the star should be above the threshold density permitting these processes to occur, and the star would have to be much more massive. Such alternative reactions are thus disfavored by the data.

10.1103/Physics.11.42 